\title{
Decoherence of quantum parameter estimation for open Dirac particle in Garfinkle-Horowitz-Strominger dilation black hole
}

\author{
Yumeng Huang ${ }^{1}$, Kai Yan ${ }^{1}$, Yinzhong Wu $\mathbf{u}^{1}$, Xiang Hao ${ }^{1,2, a}$ \\ ${ }^{1}$ School of Mathematics and Physics, Suzhou University of Science and Technology, Suzhou 215009, Jiangsu, People's Republic of China \\ ${ }^{2}$ Pacific Institute of Theoretical Physics, Department of Physics and Astronomy, University of British Columbia, 6224 Agriculture Rd., Vancouver, \\ BC V6T 1Z1, Canada
}

Received: 27 January 2019 / Accepted: 17 November 2019 / Published online: 27 November 2019

(C) The Author(s) 2019

\begin{abstract}
We introduce and study a quantum channel that arises from the structure of the vacuum state of Dirac fields propagating in a Garfinkle-Horowitz-Strominger (GHS) dilation black hole spacetime. We put forward the concept of quantum information divergence, which is a new measure for relativistic parameter estimation. We employ quantum metrology to estimate the amplitude and relative phase of a Dirac field state using the quantum Fisher information and information divergence. The decoherence of quantum parameter estimation is studied through the evolution of the Bloch vector for arbitrary initial states subjected to the quantum channel and external noises. We find that the quantum information divergence decreases more than the quantum Fisher information as a function of the radiation temperature. Due to the Pauli exclusion principle and Dirac statistics, the estimation precision will gradually decrease to a non-zero value. In order to study the decoherence in the dilation black hole, we obtain the monotonic decrease of quantum coherence when an initial field evolves from the highly correlated state to the current cosmic background. The external noises can further suppress the decoherence effect from the black hole.
\end{abstract}

\section{Introduction}

In recent years, the concepts developed in statistical mechanics and quantum foundations have been used to understand quantum effects of spacetime [1-4]. The ideas of quantum information theory such as quantum entanglement shed new light on some well known effects including Unruh acceleration effects [5,6], Gibbons-Hawking effects [7,8], and Hawking radiation from the black hole $[9,10]$. It is interesting that the spacetime with a horizon can prevent an observer from having access to the full quantum state of the field. It can result in the information loss in curved spacetime $[11,12]$. In some proposals [13-17], curved spacetime can be simulated by a system of coupled atoms and quantum fields. Quantum parameter estimation in these simulated experiments can be exploited to study quantum effects of black holes. The combination of relativity and quantum measurement can contribute to the development of relativistic quantum metrology [1825]. In some previous works [19-24], relativistic effects on quantum parameter estimation have been computed using the quantum Fisher and skew information [26-28]. Meanwhile, it is worth noticing that information divergence or relative entropy [29-31] can be used for the discrimination of quantum states.

Enlightened by that, we put forward the concept of quantum information divergence to evaluate quantum parameter in the relativistic condition. Moreover, we use the theory of open quantum systems to characterize the vacuum structure for Dirac field mode in the GHS dilation black hole. The quantum Fisher information and information divergence can be used to evaluate the amplitude and relative phase of Dirac particle. In this case, one observer freely falls toward the GHS dilation black hole and then hovers near the event horizon. The detector of the observer can detect Dirac particle.

The paper is organized as follows. In Sect. 2, the vacuum structure for Dirac fields in the GHS dilation black hole is characterized by the quantum channel with dilation parameters. We define quantum information divergence in order to quantify the parameter estimation in Sect. 3. We also investigate the dynamical behavior of quantum parameter estimation and quantum coherence. In Sect. 4, we analyze the relation of quantum coherence and quantum parameter estimation. The impacts of another external noises are taken into account.

\footnotetext{
a e-mail: xhao@mail.usts.edu.cn
} 


\section{Quantum channel of the vacuum structure for Dirac fields in the GHS dilation black hole}

In GHS dilation black hole [32], the thermal Fermi-Dirac distribution of particles with the Hawking temperature $T=$ $\frac{1}{8 \pi(M-D)}$ has been computed [7,9]. The presence of such radiation is known as the Hawking effect. The cosmological parameters $M$ and $D$ are related to the mass of black hole and dilation field. With respect to the Dirac vacuum field in GHS dilation black hole, we choose the global coordinates $(t, r, \theta, \phi)$ to express the spherically symmetric line element of the GHS black hole as [33-35],

$$
\begin{aligned}
\mathrm{d} s^{2}= & -\left(\frac{r-2 M}{r-2 D}\right) \mathrm{d} t^{2}+\left(\frac{r-2 M}{r-2 D}\right)^{-1} \mathrm{~d} r^{2} \\
& +r(r-2 D)\left(\mathrm{d} \theta^{2}+\sin ^{2} \theta \mathrm{d} \phi^{2}\right),
\end{aligned}
$$

where the natural units $\hbar=G=c=\kappa_{B}=1$ are used. The Dirac equation has the expression of $\gamma^{\alpha} e_{a}^{\mu}\left(\partial_{\mu}+\Gamma_{\mu}\right) \psi=0$ [33]. Here, $\gamma^{\alpha}$ is the Dirac matrix, $e_{a}^{\mu}$ corresponds to the inverse of the tetrad $e_{a}^{\mu}$ and $\Gamma_{\mu}$ denotes the spin connection coefficient. By solving the Dirac equation, we can obtain the positive frequency outgoing solutions outside region $I$ and inside region $I I$ of the event horizon, $\psi_{k}^{l+}=\Re e^{\mp i \omega u}$ where $\iota=(I, I I)$ represents two regions, $\boldsymbol{k}$ denotes a variable that labels the field mode, $\Re$ is a 4-component Dirac spinor and $\omega$ is a monochromatic frequency of the Dirac field. $u=$ $t-2(M-D) \ln [(r-2 M) /(2 M-2 D)]$ is the retarded time. To describe the causal structure of the GHS dilation black hole, we plot the Carter-Penrose diagrams for this spacetimes in Fig. 1. This black hole has the singularity $r=2 D$. The event horizon is given by $r=2 M$. The diagram contains two regions $I$ and $I I$ that are causally disconnected.

By using the complete orthogonal basis $\psi_{k}^{\iota \pm}$, we quantize the Dirac field as

$\psi_{\text {out }}=\sum_{\iota=I, I I} \int \mathrm{d} \boldsymbol{k}\left(a_{\boldsymbol{k}}^{\iota} \psi_{\boldsymbol{k}}^{\iota+}+b_{\boldsymbol{k}}^{\iota *} \psi_{\boldsymbol{k}}^{l-}\right)$,

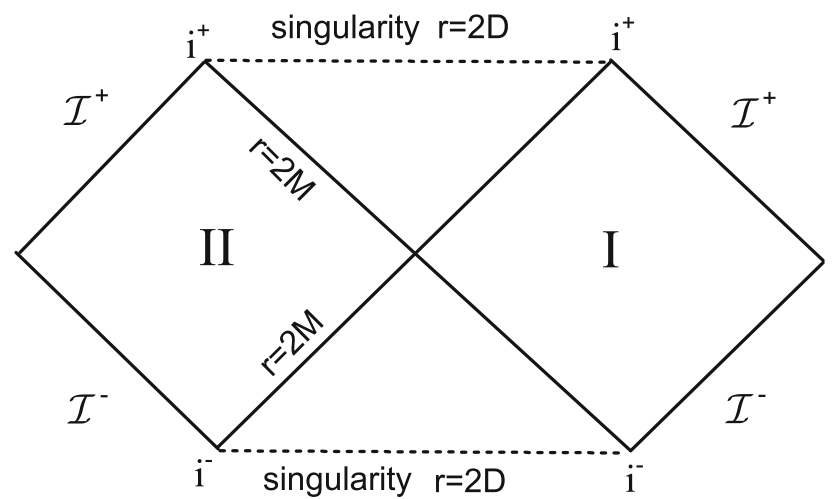

Fig. 1 The Penrose diagrams for the GHS dilation black hole can be illustrated where $a_{k}^{l}$ and $b_{k}^{\iota *}$ correspond to the fermion annihilation and antifermion creation operators respectively. The generalized Kruskal coordinates are utilized to obtain another kind of complete orthogonal basis for the positive energy mode as $\chi_{\boldsymbol{k}}^{I+}=e^{2(M-D) \pi \omega} \psi_{\boldsymbol{k}}^{I+}+e^{-2(M-D) \pi \omega} \psi_{-\boldsymbol{k}}^{I I-}, \chi_{\boldsymbol{k}}^{I I+}=$ $e^{-2(M-D) \pi \omega} \psi_{-\boldsymbol{k}}^{I-}+e^{2(M-D) \pi \omega} \psi_{\boldsymbol{k}}^{I I+}$.

Considering them as new bases, we can expand the Dirac fields in the Kruskal coordinates

$$
\begin{aligned}
\psi_{\text {out }}= & \sum_{\iota=I, I I} \int \mathrm{d} \boldsymbol{k} \frac{1}{\sqrt{2 \cosh [4(M-D) \pi \omega]}} \\
& \times\left(c_{k}^{l} \chi_{k}^{\iota+}+d_{k}^{l *} \chi_{k}^{\iota-}\right),
\end{aligned}
$$

where $c_{k}^{l}$ and $d_{k}^{l *}$ correspond to the fermion annihilation and antifermion creation operators acting on the Kruskal vacuum. The Eqs. 2 and 3 correspond to the decomposition of the Dirac fields in GHS dilation and Kruskal modes respectively. By using the appropriate Bogoliubov transformation between two different sets of coordinates, each annihilation operator $c_{k}^{l}$ can be expanded by the spacetime operators as $c_{k}^{I}=\left(e^{-\frac{\omega}{T}}+1\right)^{-\frac{1}{2}} a_{\boldsymbol{k}}^{I}-\left(e^{\frac{\omega}{T}}+1\right)^{-\frac{1}{2}} b_{\boldsymbol{k}}^{I I *}$ where $T$ denotes the radiation temperature [36,37]. The mode of ground state in the GHS dilation coordinates is considered as a two-mode squeezed state in Kruskal coordinates. Therefore, the vacuum and only excited state of the particle can be written as

$$
\begin{aligned}
|0\rangle^{+} & =\left(e^{-\frac{\omega}{T}}+1\right)^{-\frac{1}{2}}|0\rangle_{I}^{-}|0\rangle_{I I}^{-}+\left(e^{\frac{\omega}{T}}+1\right)^{-\frac{1}{2}}|1\rangle_{I}^{+}|1\rangle_{I I}^{-}, \\
|1\rangle^{+} & =|1\rangle_{I}^{+}|0\rangle_{I I}^{-},
\end{aligned}
$$

where $|n\rangle_{I}^{+}$and $|n\rangle_{I I}^{-}$represent the orthonormal basis for the outside region $I$ and inside region $I I$ of the event horizon respectively. The superscripts on the kets $\{+,-\}$ denote the particle and antiparticle. In the following context, the notation of $|n\rangle_{I, I I}^{ \pm}$is simplified as $|n\rangle_{I, I I}$.

Since the exterior region $I$ is causally disconnected from the interior region $I I$ of the corresponding spacetime, we can obtain the physical accessible part $I$ by tracing over the state of the interior region $I I$. The whole process can be described by a quantum channel $[38,39]$. According to the dynamics of open quantum system, the state of the open system will change as a consequence of its internal dynamics and of the interaction with the environment. The inevitable coupling to the environment can result in quantum decoherence which is similar to the loss of information in the spacetimes with a horizon. The state change of the open system over time $t$ is called a dynamical map or quantum channel. The map can be expressed by $\rho(0) \mapsto \rho(t)=\boldsymbol{V}(t) \cdot \rho(0)$ where $\rho(0)$ is an initial state and $\rho(t)$ is the evolved state. In general, the mathematical expression of $\boldsymbol{V}(t)$ can be written in the form of the local operators, $\boldsymbol{V}(t) \cdot \rho(0)=\sum_{\alpha, \beta} W_{\alpha, \beta}(t) \rho(0) W_{\alpha, \beta}^{\dagger}(t)$. For an arbitrary qubit state $\rho(0)=\sum_{j, k=0,1} \quad \varrho_{j k}|j\rangle\langle k|$, we can rewrite the map as $\boldsymbol{V}(t) \cdot \rho(0)=\sum_{j, k} \boldsymbol{\varepsilon}(|j\rangle\langle k|) \varrho_{j k}$. Here, the operator $\boldsymbol{\varepsilon}(|j\rangle\langle k|)=\sum_{\alpha, \beta} W_{\alpha, \beta}(t)|j\rangle\langle k| W_{\alpha, \beta}^{\dagger}(t)$. 
In regard to GHS dilation black hole, the dynamical map is defined as the Unruh channel,

$$
\begin{aligned}
& \boldsymbol{\varepsilon}_{\mathrm{U}}(|0\rangle\langle 0|)=\left(e^{-\frac{\omega}{T}}+1\right)^{-1}|0\rangle_{I}\left\langle 0\left|+\left(e^{\frac{\omega}{T}}+1\right)^{-1}\right| 1\right\rangle_{I}\langle 1| \\
& \boldsymbol{\varepsilon}_{\mathrm{U}}(|0\rangle\langle 1|)=\left(e^{-\frac{\omega}{T}}+1\right)^{-\frac{1}{2}}|0\rangle_{I}\langle 1| \\
& \boldsymbol{\varepsilon}_{\mathrm{U}}(|1\rangle\langle 0|)=\left(e^{-\frac{\omega}{T}}+1\right)^{-\frac{1}{2}}|1\rangle_{I}\langle 0| \\
& \boldsymbol{\varepsilon}_{\mathrm{U}}(|1\rangle\langle 1|)=|1\rangle_{I}\langle 1|,
\end{aligned}
$$

where the partial trace over the state of the interior region is carried out.

For an arbitrary qubit state, the density matrix of the state $\rho$ can be expressed by the vector in four-dimensional space as

$\rho=\frac{1}{2} \sum_{\mu=0}^{3} \zeta_{\mu} \sigma_{\mu}$

where $\zeta_{\mu}=\operatorname{tr}\left(\rho \sigma_{\mu}\right), \quad(\mu=0,1,2,3)$ are four components of the state vector. $\sigma_{0}$ is the identity operator $\mathbb{I}$ and $\sigma_{i}(i=1,2,3)$ denote the three Pauli operators. By means of the quantum channel, we describe the map from the initial state vector $\boldsymbol{X}=\left(1, \zeta^{(0)}\right)$ to the evolved state vector $\boldsymbol{Y}=(1, \zeta)$ as,

$\boldsymbol{Y}=\boldsymbol{\Phi}_{\mathrm{U}} \cdot \boldsymbol{X}=\left(\begin{array}{cc}1 & \mathbf{0}^{\mathrm{T}} \\ \lambda & \Gamma\end{array}\right)\left(\begin{array}{c}1 \\ \zeta^{(0)}\end{array}\right)$,

where the vector $\mathbf{0}^{\mathrm{T}}=(0,0,0)$ and $\zeta$ is the Bloch vector of state. The quantum channel $\varepsilon_{\mathrm{U}}(\cdot)$ determines the column vector $\lambda$ with $\lambda_{j}(j=1,2,3)=\operatorname{Tr}\left[\frac{\sigma_{j}}{2} \cdot \boldsymbol{\varepsilon}_{\mathrm{U}}(\mathbb{I})\right]$. And another $3 \times 3$ acting matrix $\Gamma$ has the components which satisfy $\Gamma_{i j}(i, j=1,2,3)=\operatorname{Tr}\left[\frac{\sigma_{i}}{2} \cdot \varepsilon_{\mathrm{U}}\left(\sigma_{j}\right)\right]$. The Bloch vector of the evolved state can be obtained by $\zeta=\lambda+\Gamma \cdot \zeta^{(0)}$.

Using Eqs. (5) and (6), the $4 \times 4$ mathematical mapping matrix $\boldsymbol{\Phi}_{\mathrm{U}}$ for a Unruh channel can be written as

$\boldsymbol{\Phi}_{\mathrm{U}}=\left(\begin{array}{cccc}1 & 0 & 0 & 0 \\ 0 & \left(e^{-\frac{\omega}{T}}+1\right)^{-\frac{1}{2}} & 0 & 0 \\ 0 & 0 & \left(e^{-\frac{\omega}{T}}+1\right)^{-\frac{1}{2}} & 0 \\ -\left(e^{\frac{\omega}{T}}+1\right)^{-1} & 0 & 0 & \left(e^{-\frac{\omega}{T}}+1\right)^{-1}\end{array}\right)$,

where the mapping matrix is completely determined by the radiation temperature of the Unruh channel. In this way, we can have physical access to the state of the Dirac qubit by the mapping matrix of state vector.

\section{Quantum parameter estimation in the formalism of Bloch vector}

In the analogue experimental realization [15], the parameter estimation of quantum fields can play an important role in the study of relativistic effects. Quantum metrology has become a powerful tool for some tasks such as state discrimination [40,41] and tomography [19]. Our aim is to study the quantum effects of dilation black holes from the viewpoint of relativistic quantum metrology.

As is well known, quantum Fisher information can provide a tight bound for the precision of parameter estimation. The definition of quantum Fisher information is closely related to the Bures distance for the discrimination of two neighboring states $\rho(\theta)$ and $\varrho=\rho(\theta+\Delta \theta)$. Here $\theta$ is the estimated parameter and $\Delta \theta$ denotes the parameter variation. According to reference [40], the expression of Bures distance is given by $\mathcal{D}_{\mathrm{B}}^{2}(\rho, \varrho)=2[1-f(\rho, \varrho)]$ where $f(\rho, \varrho)=$ $\operatorname{Tr} \sqrt{\sqrt{\varrho} \rho \sqrt{\varrho}}$ is the fidelity of two quantum states. In the second-order approximation of $\Delta \theta$, the relation between quantum Fisher information and Bures distance is written as

$\mathcal{D}_{\mathrm{B}}^{2}(\rho, \varrho)=\frac{(\Delta \theta)^{2}}{4} \mathcal{F}_{\theta}$,

where $\mathcal{F}_{\theta}$ is the quantum Fisher information. It can be defined by the symmetric logarithmic derivative operator $[42,43]$, i.e., $\mathcal{F}_{\theta}=\operatorname{Tr}\left(\rho L^{2}\right)=\operatorname{Tr}\left(\partial_{\theta} \rho L\right)$. The symmetric logarithmic derivative $L$ is a Hermitian operator determined by $\partial_{\theta} \rho=\frac{1}{2}[\rho L+L \rho]$ where the notation $\partial_{\theta}(\cdot)=\frac{\partial}{\partial \theta}(\cdot)$. In the simplest case of a pure state $\rho=|\psi\rangle\langle\psi|, \mathcal{F}_{\mathrm{p}}=2 \operatorname{Tr}\left[\left(\partial_{\theta} \rho\right)^{2}\right]$. In the eigenstate space of $\rho=\sum_{m} p_{m}\left|\psi_{m}\right\rangle\left\langle\psi_{m}\right|$, quantum Fisher information can be rewritten as,

$$
\begin{aligned}
\mathcal{F}_{\theta}= & \mathcal{F}_{\mathrm{c}}+4 \sum_{m} p_{m} \mathcal{F}_{\mathrm{p}}\left(\left|\psi_{m}\right\rangle\right) \\
& -8 \sum_{m \neq n} \frac{p_{m} p_{n}}{p_{m}+p_{n}}\left|\left\langle\psi_{n} \mid \partial_{\theta} \psi_{m}\right\rangle\right|^{2},
\end{aligned}
$$

where the first term $\mathcal{F}_{\mathrm{c}}=\sum_{m} \frac{\left(\partial_{\theta} p_{m}\right)^{2}}{p_{m}}$ is the classical Fisher information.

With the idea of the divergence of parameter distribution, Kullback-Leibler distance is also introduced to distinguish two neighboring states [44]. The definition is $\mathcal{D}_{\mathrm{KL}}(\rho, \varrho)=$ $\operatorname{Tr}[\rho(\log \rho-\log \varrho)]$. In the Taylor expansion of $\Delta \theta$ to the second order which is $\log \rho(\theta)-\log \rho(\theta+\Delta \theta) \approx$ $-\left(\partial_{\theta} \log \rho\right) \Delta \theta-\frac{1}{2}\left(\partial_{\theta}^{2} \log \rho\right)(\Delta \theta)^{2}$, we then apply this to the definition of $\mathcal{D}_{\mathrm{KL}}$

$\mathcal{D}_{\mathrm{KL}}=\operatorname{Tr}\left\{\rho\left[-\left(\partial_{\theta} \log \rho\right) \Delta \theta-\frac{1}{2}\left(\partial_{\theta}^{2} \log \rho\right)(\Delta \theta)^{2}\right]\right\}$,

where $\log \rho$ can be expressed by its eigenstates as $\sum_{m} \log$ $p_{m}\left|\psi_{m}\right\rangle\left\langle\psi_{m}\right|$.

$$
\begin{aligned}
\partial_{\theta} \log \rho= & \sum_{m} \frac{1}{p_{m}} \partial_{\theta} p_{m}\left|\psi_{m}\right\rangle\left\langle\psi_{m}\right| \\
& +\sum_{m} \log p_{m}\left|\partial_{\theta} \psi_{m}\right\rangle\left\langle\psi_{m}\right| \\
& +\sum_{m} \log p_{m}\left|\psi_{m}\right\rangle\left\langle\partial_{\theta} \psi_{m}\right|,
\end{aligned}
$$


where $\left\langle\psi_{m} \mid \partial_{\theta} \psi_{m}\right\rangle+\left\langle\partial_{\theta} \psi_{m} \mid \psi_{m}\right\rangle=\partial_{\theta}\left\langle\psi_{m} \mid \psi_{m}\right\rangle=0$ and $\sum_{m} p_{m}=1$.

Then, the expression of Eq. (11) can be rewritten as

$$
\begin{aligned}
\mathcal{D}_{\mathrm{KL}}= & \frac{(\Delta \theta)^{2}}{2} \sum_{m} \frac{p_{m}}{2} \log p_{m}\left(\left\langle\partial_{\theta} \psi_{m} \mid \partial_{\theta} \psi_{m}\right\rangle-\left|\left\langle\psi_{m} \mid \partial_{\theta} \psi_{m}\right\rangle\right|^{2}\right) \\
& +\frac{(\Delta \theta)^{2}}{2} \sum_{m}\left[\frac{\left(\partial_{\theta} p_{m}\right)^{2}}{p_{m}}-2 \sum_{m \neq n} p_{n} \log p_{m}\left|\left\langle\psi_{n} \mid \partial_{\theta} \psi_{m}\right\rangle\right|^{2}\right],
\end{aligned}
$$

where the relation of $\partial_{\theta}^{2}\left\langle\psi_{m} \mid \psi_{m}\right\rangle=\left\langle\partial_{\theta}^{2} \psi_{m} \mid \psi_{m}\right\rangle+\left\langle\psi_{m} \mid \partial_{\theta}^{2} \psi_{i}\right\rangle$ $+2\left\langle\partial_{\theta} \psi_{m} \mid \partial_{\theta} \psi_{m}\right\rangle=0$ is used.

Similar to Eq. (9), we can define a quantity $\mathcal{G}(\theta)$ to express the Kullback-Leibler distance

$\mathcal{D}_{\mathrm{KL}}(\rho, \varrho)=\frac{(\Delta \theta)^{2}}{2} \mathcal{G}_{\theta}$,

where $\mathcal{G}_{\theta}$ is referred to as quantum information divergence. The expression of $\mathcal{G}_{\theta}$ can be given by

$$
\begin{aligned}
\mathcal{G}_{\theta}= & \mathcal{F}_{\mathrm{c}}+\frac{1}{2} \sum_{m} p_{m} \log p_{m} \mathcal{F}_{\mathrm{p}}\left(\left|\psi_{m}\right\rangle\right) \\
& -2 \sum_{m \neq n} p_{n} \log p_{m}\left|\left\langle\psi_{n} \mid \partial_{\theta} \psi_{m}\right\rangle\right|^{2},
\end{aligned}
$$

where the second term results from the quantum Fisher information of all pure states $\left|\psi_{m}\right\rangle$ and the third term results from the mixture of all eigenstates. In a sense, Eqs. (14) and (15) can provide an evidence that there is the close relationship between quantum Fisher information and information divergence. For a pure state, $\mathcal{G}_{\theta}=\infty$ and $\mathcal{F}_{\theta}=1$. And $\mathcal{G}_{\theta}=\mathcal{F}_{\theta}=0$ for complete mixed states. As we know, the evolved state of an open quantum system will be mixed if the quantum noise channel acts on an arbitrary initial state. Therefore, quantum information divergence $\mathcal{G}$ can be used to estimate the parameter for a general mixed state of an open system.

In the formalism of state vector, we can rewrite $\mathcal{F}_{\theta}$ and $\mathcal{G}_{\theta}$ as

$$
\begin{aligned}
& \mathcal{F}_{\theta}=\left|\partial_{\theta} \zeta\right|^{2}+\frac{\left(\zeta \cdot \partial_{\theta} \zeta\right)^{2}}{1-|\zeta|^{2}} \\
& \mathcal{G}_{\theta}=\frac{\left|\partial_{\theta} \zeta\right|^{2}-\left(\partial_{\theta}|\zeta|\right)^{2}}{|\zeta|} \log \frac{1+|\zeta|}{1-|\zeta|}+\frac{\left(\partial_{\theta}|\zeta|\right)^{2}}{1-|\zeta|^{2}}
\end{aligned}
$$

where $\zeta$ denotes the Bloch vector of a Dirac qubit.

We consider a Unruh channel acting on a Dirac field qubit with an initial state $|\psi\rangle=\cos \frac{\alpha}{2}|1\rangle+e^{i \beta} \sin \frac{\alpha}{2}|0\rangle . \alpha \in[0, \pi)$ and $\beta \in[0,2 \pi)$ represent the amplitude and phase parameter of the Dirac field respectively. Here we use the coordinates of the GHS dilation black hole to represent the vacuum state $|0\rangle$ and excited one $|1\rangle$. In a sense, $\cos ^{2} \frac{\alpha}{2}$ and $\sin ^{2} \frac{\alpha}{2}$ correspond to the survival probabilities of the vacuum state and excited one during the evolution of the dilation black hole. According to Eq. (5), we find that there is the oscillation probability between the vacuum state and excited one in the physical accessible part $I$. The process of the oscillation can give rise to the relative phase $\beta$. The Bloch vector of the initial state is expressed as $\zeta^{(0)}=(\cos \beta \sin \alpha, \sin \beta \sin \alpha, \cos \alpha)^{\mathrm{T}}$. Using Eq. (16), we can obtain the quantum parameter estimation about $\alpha$ and $\beta$ as

$\mathcal{F}_{\alpha}=\cos ^{2} r$

$\mathcal{F}_{\beta}=\cos ^{2} r \sin ^{2} \alpha$,

$\mathcal{G}_{\alpha}=\mathcal{F}_{\alpha}\left(\frac{|\zeta|^{2}-\sin ^{2} r \sin ^{2} \alpha}{|\zeta|^{3}} \log \frac{1+|\zeta|}{1-|\zeta|}+\frac{\sin ^{2} r \sin ^{2} \alpha}{|\zeta|^{2}}\right)$,

$\mathcal{G}_{\beta}=\frac{\mathcal{F}_{\beta}}{|\zeta|} \log \frac{1+|\zeta|}{1-|\zeta|}$

where $|\zeta|=\sqrt{1-\cos ^{2} r \sin ^{2} r(1+\cos \alpha)^{2}}$ and $\cos r=$ $\left(e^{-\frac{\omega}{T}}+1\right)^{-\frac{1}{2}}$. In the following analysis, we exploit the vector form of quantum parameter estimation to study the decoherence.

\section{Decoherence without and with external noises}

The dynamics of the Dirac field state in GHS dilation black hole can be governed by the Unruh channel with the radiation temperature $T$. The change of $\mathcal{G}_{\alpha}$ can be plotted as a function of radiation temperature $T$ in Fig. 2. According to Eq. (17), $\mathcal{F}_{\alpha}$ is independent of the initial Dirac field state and only determined by the radiation temperature of the curved spacetime. Different from $\mathcal{F}_{\alpha}$, the quantum information divergence $\mathcal{G}_{\alpha}$ decreases from the infinity value to a fixed value. In Fig. 2 , we illustrate the asymmetric behavior of the quantum information divergence with respect to $\alpha$. From Fig. 3, we can see that both of estimation values are monotonically decreased to non-zero steady values with the increase of temperature. It results from the Pauli exclusion principle and Fermi statistics of Dirac fields.

Meanwhile, the estimation precision of parameter $\beta$ can also be performed by the measures of $\mathcal{F}_{\beta}$ and $\mathcal{G}_{\beta}$. From Eq. (17), we can see that quantum Fisher information $\mathcal{F}_{\beta}$ is symmetric about $\alpha$. The maximum value of $\mathcal{F}_{\beta}$ is obtained when the maximally coherent initial state with $\alpha=\pi / 2$ is chosen. $\mathcal{G}_{\alpha}$ is plotted as a function of $\alpha$ and $T$ in Fig. 4. Similar to quantum Fisher information, the maximum value of $\mathcal{G}_{\alpha}$ arrives at $\alpha=\pi / 2$. This phenomenon can demonstrate that quantum information divergence is considered as another efficient measure for quantum parameter estimation.

To explain the mechanism of the decoherence, we further calculate the quantum coherence of the Dirac field in the Unruh-channel spacetime. The relative entropy of coherence 


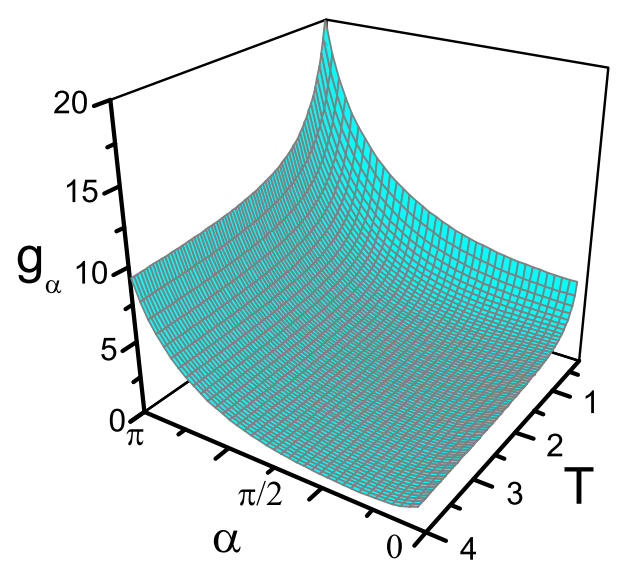

Fig. 2 The quantum parameter estimation with respect to $\alpha$ is evaluated by $\mathcal{G}_{\alpha}$ as a function of radiation temperature $T$ when the frequency of Dirac field $\omega=1$ is chosen

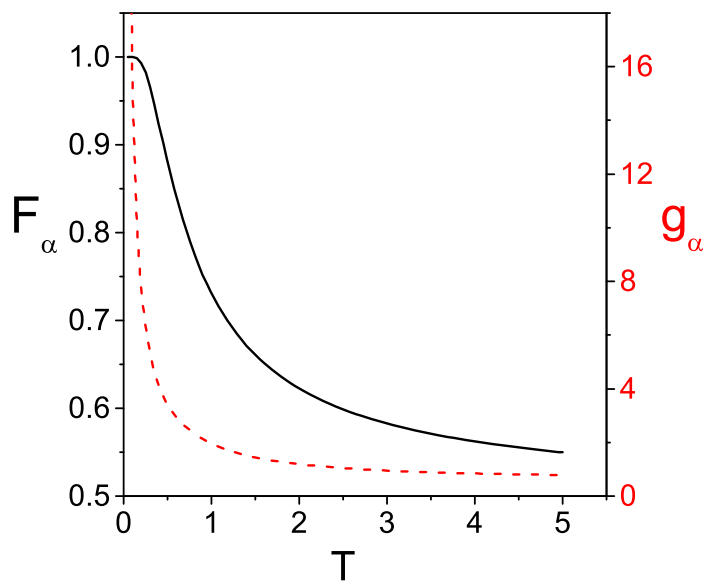

Fig. 3 The decoherence of $\mathcal{F}_{\alpha}$ and $\mathcal{G}_{\alpha}$ is illustrated by the black line and red dash line respectively. The parameters of $\alpha=\pi / 4$ and $\omega=1$ are chosen

[45] is introduced as $C(\rho)=S\left(\rho_{\text {diag }}\right)-S(\rho)$ where the von Neumann entropy $S(\rho)=-\operatorname{Tr}[\rho \log (\rho)]$ and $\rho_{\text {diag }}$ denotes the state which is obtained from $\rho$ by deleting all off-diagonal elements. For any Dirac qubit, quantum coherence is written as

$C(\rho)=1+|\zeta| \log |\zeta|$,

where $\zeta$ is the Bloch vector.

When the initial state $|\psi\rangle=\cos \frac{\alpha}{2}|1\rangle+e^{i \beta} \sin \frac{\alpha}{2}|0\rangle$ is chosen, the changes of quantum coherence is numerically illustrated in Fig. 5. It is clearly shown that quantum coherence keeps a high value at the early stage and then decays gradually to a non-zero steady value. The behaviour of quantum coherence is related to the transition of quantum effects of Dirac fields. The increase in the radiation temperature can give rise to the transition from a high correlated state to a mixed steady state with less coherence. This decoherence

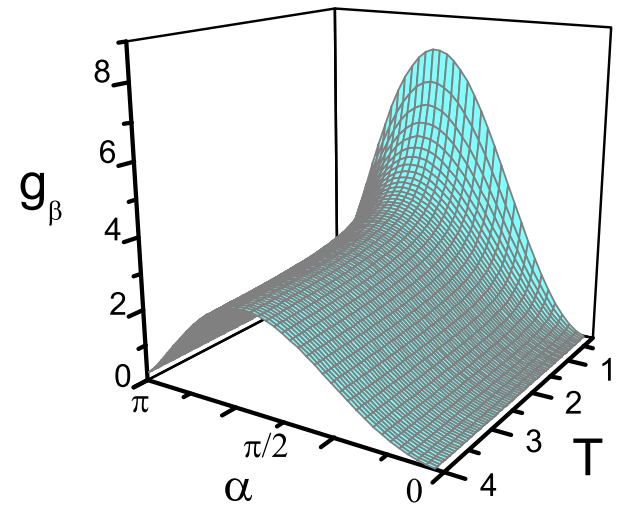

Fig. 4 The quantum parameter estimation with respect to $\beta$ is evaluated by $\mathcal{G}_{\beta}$ as a function of the amplitude $\alpha$ and radiation temperature $T$ when the frequency of Dirac field $\omega=1$ is chosen

phenomenon of quantum coherence is very similar to that of quantum parameter estimation.

Additionally, it is interesting to analyze the effects of external noises on a Dirac qubit in the Unruh channel. Under the influence of ambient effects and cosmological radiation, the behavior of quantum parameter estimation can contribute to the deep understanding of quantum effects of spacetime. For this purpose, we consider two typical kinds of noises which are described by an amplitude damping channel (AD) and phase damping channel (PD) [46]. Here, we exploit the quantum channel to construct the evolution of Dirac state with external noises.

In general, when a series of quantum channels $\left\{\boldsymbol{\varepsilon}_{i}, i=\right.$ $1,2, \ldots, N\}$ sequentially act on an initial state $\rho^{(0)}$, the evolved state $\rho$ can be governed by $\rho=\boldsymbol{\varepsilon}_{N} \cdot \boldsymbol{\varepsilon}_{N-1} \cdots \boldsymbol{\varepsilon}_{1}\left(\rho^{(0)}\right)$. For the simplification of the expression, we assume the notation $\Pi_{i=1}^{N} \boldsymbol{\varepsilon}_{i}=\boldsymbol{\varepsilon}_{N} \cdot \boldsymbol{\varepsilon}_{N-1} \cdots \boldsymbol{\varepsilon}_{1}$. If each channel is expressed in the form of mapping matrix $\boldsymbol{\Phi}_{i}$, the state vector during the evolution can be obtained by

$\boldsymbol{Y}=\Pi_{i=1}^{N} \boldsymbol{\Phi}_{i} \cdot \boldsymbol{X}$

where the product of these mapping matrices can be expressed by a total mapping matrix $\boldsymbol{\Phi}_{\text {tot }}$ as

$\boldsymbol{\Phi}_{\text {tot }}=\Pi_{i=1}^{N} \boldsymbol{\Phi}_{i}=\left(\begin{array}{cc}1 & \mathbf{0}^{\mathrm{T}} \\ \lambda_{\mathrm{tot}} & \boldsymbol{\Gamma}_{\text {tot }}\end{array}\right)$.

Thus, the total evolution process is equivalent to the total mapping matrix with the effective vector $\lambda_{\text {tot }}=\lambda_{N}+$ $\sum_{j=2}^{N}\left[\left(\Pi_{k=j}^{N} \boldsymbol{\Gamma}_{k}\right) \lambda_{j-1}\right]$ and acting matrix $\boldsymbol{\Gamma}_{\text {tot }}=\Pi_{i=1}^{N} \boldsymbol{\Gamma}_{i}$. The Bloch vector of the evolved state can be obtained by $\zeta=\lambda_{\text {tot }}+\Gamma_{\text {tot }} \cdot \zeta^{(0)}$.

In the simplest case of $N=2$, an open Dirac qubit are subjected to the Unruh channel $\boldsymbol{\Phi}_{\mathrm{U}}$ and other noisy channel 


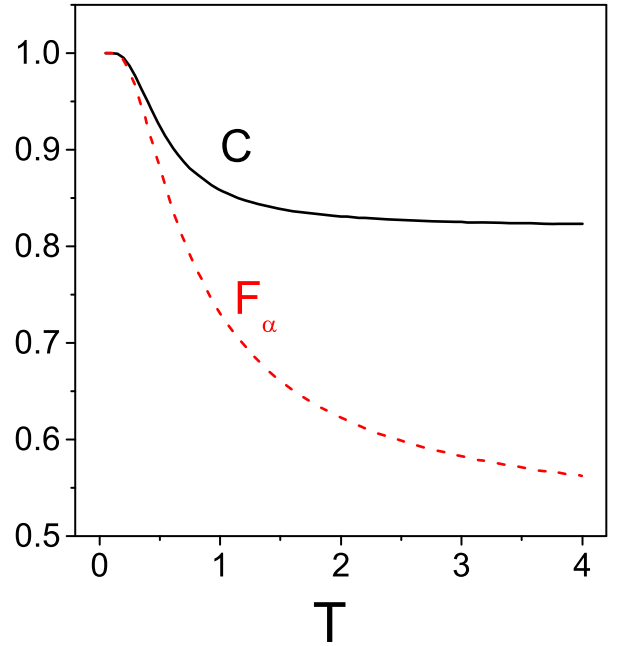

Fig. 5 The decoherence of quantum coherence $C$ and quantum Fisher information $\mathcal{F}_{\alpha}$ are illustrated by the black line and red dash line respectively. The parameters of $\alpha=\pi / 2$ and $\omega=1$ are chosen

of $\boldsymbol{\Phi}_{\mathrm{AD}}, \boldsymbol{\Phi}_{\mathrm{PD}}$. In both cases, the mapping matrices can be obtained by

$\Phi^{(1)}=\left(\begin{array}{cccc}1 & 0 & 0 & 0 \\ 0 & \sqrt{1-p} \cos r & 0 & 0 \\ 0 & 0 & \sqrt{1-p} \cos r & 0 \\ (1-p) \cos ^{2} r-1 & 0 & 0 & (1-p) \cos ^{2} r\end{array}\right)$,

$\Phi^{(2)}=\left(\begin{array}{cccc}1 & 0 & 0 & 0 \\ 0 & (1-p) \cos r & 0 & 0 \\ 0 & 0 & (1-p) \cos r & 0 \\ -\sin ^{2} r & 0 & 0 & \cos ^{2} r\end{array}\right)$,

where $\boldsymbol{\Phi}^{(1)}$ for the amplitude damping case and $\boldsymbol{\Phi}^{(2)}$ for the phase damping one. The parameter $p \in[0,1)$ denotes the decaying coefficient from the environmental noise. $p=$ 0 represents the Unruh channel without any noise. In both of cases, the Bloch vectors $\zeta^{(i)}$ for the Dirac state can be calculated as

$\zeta^{(1)}=\left(\begin{array}{c}\sqrt{1-p} \cos r \cos \beta \sin \alpha \\ \sqrt{1-p} \cos r \sin \beta \sin \alpha \\ (1-p) \cos ^{2} r \cos \alpha-p \cos ^{2} r-\sin ^{2} r\end{array}\right)$,

$\zeta^{(2)}=\left(\begin{array}{c}(1-p) \cos r \cos \beta \sin \alpha \\ (1-p) \cos r \sin \beta \sin \alpha \\ \cos ^{2} r \cos \alpha-\sin ^{2} r\end{array}\right)$,

which can be used to evaluate the quantum parameter estimation according to Eq. (16). For example, quantum parameter estimation with respect to $\beta$ are obtained by means of two measures,

$\mathcal{F}_{\beta}^{(1)}=(1-p) \mathcal{F}_{\beta}$,

$\mathcal{F}_{\beta}^{(2)}=(1-p)^{2} \mathcal{F}_{\beta}$,

$\mathcal{G}_{\beta}^{(1)}=\mathcal{F}_{\beta} \frac{(1-p)}{\left|\zeta^{(1)}\right|} \log \frac{1+\left|\zeta^{(1)}\right|}{1-\left|\zeta^{(1)}\right|}$,
$\mathcal{G}_{\beta}^{(2)}=\mathcal{F}_{\beta} \frac{(1-p)^{2}}{\left|\zeta^{(2)}\right|} \log \frac{1+\left|\zeta^{(2)}\right|}{1-\left|\zeta^{(2)}\right|}$

where $\mathcal{F}_{\beta}$ is the quantum Fisher information for the Unruh channel without external noises. From the above analytical results, it is found out that the estimation precision can be further suppressed by the external noises.

\section{Discussion}

We exploit the theory of quantum channels to study the structure of the vacuum state of Dirac fields propagating in a GHS dilation black hole spacetime. We introduce the concept of quantum information divergence for relativistic parameter estimation. We use the quantum Fisher information and information divergence to estimate the amplitude and relative phase of the Dirac field state. The precision of estimating the phase parameter can decrease with the radiation temperature. The external noise from the amplitude damping channel and phase damping channel can suppress the decoherence of quantum parameter estimation.

Acknowledgements We would like to thank Professor Bill Unruh and Professor Philip C. E. Stamp for the discussions on related work. This work is funded by $\mathrm{SCOAP}^{3}$.

Data Availability Statement This manuscript has no associated data or the data will not be deposited. [Authors' comment: The manuscript has no associated data.]

Open Access This article is distributed under the terms of the Creative Commons Attribution 4.0 International License (http://creativecomm ons.org/licenses/by/4.0/), which permits unrestricted use, distribution, and reproduction in any medium, provided you give appropriate credit to the original author(s) and the source, provide a link to the Creative Commons license, and indicate if changes were made. Funded by $\mathrm{SCOAP}^{3}$.

\section{References}

1. R. Müller, C.O. Lousto, Phys. Rev. D 52, 4512 (1995)

2. A. Peres, D.R. Terno, Rev. Mod. Phys. 76, 93 (2004)

3. P.M. Alsing, I. Fuentes-Schuller, R.B. Mann, T.E. Tessier, Phys. Rev. A 74, 032326 (2006)

4. E. Martín-Martínez, N.C. Menicucci, Class. Quantum Gravity 29, 224003 (2012)

5. W.G. Unruh, Phys. Rev. D 14, 870 (1976)

6. L.C.B. Crispino, A. Higuchi, G.E.A. Matsas, Rev. Mod. Phys. 80, 787 (2008)

7. G.W. Gibbons, S.W. Hawking, Phys. Rev. D 15, 2738 (1977)

8. H. Yu, Phys. Rev. Lett. 106, 061101 (2011)

9. S.W. Hawking, Commun. Math. Phys. 43, 199 (1975)

10. J. He, S. Xu, L. Ye, Phys. Lett. B 756, 278 (2016)

11. M. Franco, E. Calzetta, Class. Quantum Gravity 28, 145024 (2011)

12. S. Banerjee, A.K. Alok, S. Omkar, Eur. Phys. J. C. 76, 437 (2016)

13. U.R. Fischer, R. Schützhold, Phys. Rev. A 70, 063615 (2004)

14. N.C. Menicucci, S.J. Olson, G.J. Milburn, New. J. Phys. 12, 095019 (2010) 
15. M. Aspachs, G. Adesso, I. Fuentes, Phys. Rev. Lett. 105, 151301 (2010)

16. P.D. Nation, J.R. Johansson, M.P. Blencowe, F. Nori, Rev. Mod. Phys. 84, 1 (2012)

17. N. Friis, M. Huber, I. Fuentes, D.E. Bruschi, Phys. Rev. D 86, 105003 (2012)

18. D. Hosler, P. Kok, Phys. Rev. A 88, 052112 (2013)

19. Y. Yao, X. Xiao, L. Ge, X. Wang, C. Sun, Phys. Rev. A 89, 042336 (2014)

20. M. Ahmadi, D.E. Bruschi, N. Friis, C. Sabín, G. Adesso, I. Fuentes, Sci. Rep. 4, 4996 (2014)

21. Z. Tian, J. Wang, H. Fan, J. Jing, Sci. Rep. 5, 7946 (2015)

22. X. Hao, Y.Z. Wu, Ann. Phys. 372, 110 (2016)

23. J. Kohlrus, D.E. Bruschi, J. Louko, I. Fuentes, EPJ Quantum Technol. 4, 7 (2017)

24. Z.H. Tian, J.C. Wang, J.L. Jing, A. Dragan, Ann. Phys. 377, 1 (2017)

25. R. Howl, L. Hackermueller, D.E. Bruschi, I. Fuentes, Adv. Phys. X 3, 1383184 (2018)

26. B.R. Frieden, Physics from Fisher Information: A Unification (Cambridge University Press, Cambridge, 1998)

27. S. Luo, Phys. Rev. Lett. 91, 180403 (2003)

28. G. Girolami, Phys. Rev. Lett. 113, 170401 (2014)

29. S. Kullback, Information Theory and Statistics (Wiley, Hoboken, 1959)
30. V. Vedral, Rev. Mod. Phys. 74, 197 (2002)

31. A. Winter, Commun. Math. Phys. 347, 291 (2016)

32. T. Damoar, R. Ruffini, Phys. Rev. D 14, 332 (1976)

33. J. Wang, Q. Pan, J. Jing, Ann. Phys. 325, 1190 (2010)

34. D. Garfinkle, G.T. Horowitz, A. Strominger, Phys. Rev. D 43, 3140 (1991)

35. A. Gareia, D. Galtsov, O. Kechkin, Phys. Rev. Lett. 74, 1276 (1995)

36. E. Martín-Martínez, L.J. Garay, J. León, Phys. Rev. D 82, 064006 (2010)

37. D.E. Bruschi, J. Louko, E. Martín-Martínez, A. Dragan, I. Fuentes, Phys. Rev. A 82, 042332 (2010)

38. M.O. Scully, M.S. Zubairy, Quantum Optics (Cambridge University Press, Cambridge, 1997)

39. S.M. Barnett, P.M. Radmore, Methods in Theoretical Quantum Optics (Oxford University Press, Oxford, 1997)

40. S.L. Braunstein, C.M. Caves, Phys. Rev. Lett. 72, 3439 (1994)

41. A. Chefles, Contemp. Phys. 41, 401 (2000)

42. C.W. Helstrom, Quantum Detection and Estimation Theory (Academic, New York, 1976)

43. A.S. Holevo, Probabilistic and Statistical Aspects of Quantum Theory (North-Holland, Amsterdam, 1982)

44. Fumio Hiai, Dénes Petz, Commun. Math. Phys. 143, 99 (1991)

45. T. Baumgratz, M. Cramer, M.B. Plenio, Phys. Rev. Lett. 113, $140401(2014)$

46. M.A. Nielsen, I.L. Chuang, Quantum Computation and Quantum Information (Cambridge University Press, Cambridge, 2011) 